Proyecciones

Vol. 17, No 2, pp. 215-226, December 1998

Universidad Católica del Norte

Antofagasta - Chile

\title{
ARISING OF BERGMAN REPRODUCING KERNEL IN BOSE-FOCK SPACE
}

\author{
OLE RASK \\ Universidad Austral de Chile, Valdivia - Chile.
}

\begin{abstract}
We introduce a Bose-Fock space $\mathcal{A}\left(D^{d}\right)$ of anti-holomorphic complexvalued functions on the unit polydisc $D^{d}$ in $C^{d}$, with $d \in N \cup\{\infty\}$. A unitary isomorphism $U$ from the abstract Bose-Fock space $\Gamma \mathcal{H}$ to $\mathcal{A}\left(D^{d}\right)$ is produced, and it is shown that $U$ transforms twisted coherent vectors into Bergman kernels in $D^{d}$.
\end{abstract}




\section{Introduction}

The traditional Fock space representations as e.g. the Schrödinger reprosentation or the Scgal-Bargmann analytic function representation exist as subspaces of $L^{2}$-spaces over infinite measure spaces. On the other hand. spaces of integrable functions over finite measure regions were traditionally examined in mathematics, e.g. Hardy and Bergnian spaces. It secms natural to find out, how Bose-Fock spaces can be represented as spaces of functions on domains of finite measurc.

Some aspects of this question are exposed in [1] and [4]. The approach of this paper is clifferent. The model is inspired by the complex waverepresentation (cf. [3]). Wc introduce a Bose-Fock algebra $\mathcal{A}_{0}\left(D^{d}\right)$ of antiholomorphic complex-valued functions on the unit polydisc in $C^{d}$. Then we produce a isometric nap $U$ from the abstract Bose-Fock algebra $\Gamma_{0} \mathcal{H}$ to $\mathcal{A}_{0}\left(D^{d}\right)$. This map is extended to a unitary isomorphism $U$ from the BoseFock space $\Gamma \mathcal{H}$ to $\mathcal{A}\left(D^{d}\right)$. Eventually we see that $U$ transforns twisted coherent vectors into Bergman kernels in $D^{d}$.

\section{Operators on the algebra $\mathcal{A}_{0}\left(D^{d}\right)$}

In this paper most results are valid in both finite and infinite dimensions. We shall thus let $d$ denote the dimension, where $d \in N \cup\{\infty\}$. Unless the opposite is explicitly noticed, we shall always allow $d$ to be infinite.

Let

$$
C_{0}^{d}=\left\{\left(x_{1}, x_{2}, \ldots\right) \in C^{d}, x_{n}=0 \text { for almost cvery } n \in N_{d}\right\},
$$

where $N_{d}=\{n \in N \mid n<d+1\}$, and consider the sesquilinear scalar product $\langle x, y\rangle=\sum_{n=1}^{d} \overline{x_{n}} y_{n}$, whicre $x \in C_{0}^{d}$ and $y \in C^{d}$. Let, in the sequel, $\left\{e_{n}\right\}_{n=1}^{d}$ be the orthonormal basis in $C_{0}^{d}$ with $e_{n}=(0, \ldots, 0,1,0, \ldots) \in C_{0}^{d}$, where 1 occurs on the $n$ 'th place. Consider the polydiscs

$$
D^{d}=\left\{z \in C^{d}|| z_{n} \mid<1, \text { where } z_{n}=\left\langle e_{n}, z\right\rangle \text { and } n \in N_{d}\right\}
$$

and

$$
\overline{D^{d}}=\left\{z \in C^{d}|| z_{n} \mid \leq 1 \text {, where } z_{n}=\left\langle e_{n}, z\right\rangle \text { and } n \in N_{d}\right\} .
$$

We provide $\overline{D^{d}}$ with a probability measure, which is the (finite or infinitc) product of the normalized Lebesgue measurc on $\overline{D^{1}}$. Notice that 
$\overline{D^{d}} \backslash D^{d}$ is a set of measure zero. We write $L^{2}\left(D^{d}\right),\langle\cdot, \cdot\rangle_{2}$ for the corresponding $L^{2}$-space.

Let $\mathcal{A}_{0}\left(D^{d}\right) \subset L^{2}\left(D^{d}\right)$ be the linear space of anti-holomorphic tame polynomials (i.e. each dependent on only finitely many variables) on $D^{d}$, and denote by $\mathcal{A}\left(D^{d}\right)$ the closure of $\mathcal{A}_{0}\left(D^{d}\right)$ in $L^{2}\left(D^{d}\right)$.

Set

$$
\left(\partial_{x} f\right)(\cdot)=\left.\frac{d}{d t} f(\cdot+t x)\right|_{t=0},
$$

where $x \in C_{0}^{d}, t \in R$ and $f(\cdot) \in \mathcal{A}_{0}\left(D^{d}\right)$. We will use the operator of multiplication $\mu_{x}$, where

$$
\left(\mu_{x} f\right)(\cdot)=\langle\cdot, x\rangle f(\cdot)
$$

for $x \in C_{0}^{d}$ and $f \in \mathcal{A}_{0}\left(D^{d}\right)$.

We have the following straightforward lemma.

Lemma 1 : Given $x, y, u, v \in \mathbf{C}_{0}^{d}$, we have on $\mathcal{A}_{0}\left(\mathbf{D}^{d}\right)$ the identities

$$
\begin{gathered}
{\left[\partial_{x}, \mu_{y}\right]=\langle x, y\rangle I} \\
{\left[\partial_{x}, \partial_{y}\right]=\left[\mu_{x}, \mu_{y}\right]=0}
\end{gathered}
$$

and

$$
\left[\partial_{x}, \mu_{f}\right]=\left(\partial_{x} f\right),
$$

where $\mu_{f} g=f g$ for $f, g \in \mathcal{A}_{0}\left(\mathbf{D}^{d}\right)$.

The partial number operator $N_{k}: \mathcal{A}_{0}\left(D^{d}\right) \rightarrow \mathcal{A}_{0}\left(D^{d}\right)$ assumes the form

$$
N_{k}=\mu_{e_{k}} \partial_{e_{k}} .
$$

We consider on $\mathcal{A}_{0}\left(D^{d}\right)$ the number operator $N=\sum_{p=1}^{d} N_{p}$, with the property of $N\left\langle\cdot, x_{1}\right\rangle \cdots\left\langle\cdot, x_{m}\right\rangle=m\left\langle\cdot, x_{1}\right\rangle \cdots\left\langle\cdot, x_{m}\right\rangle$, for $x_{1}, \ldots, x_{m} \in C_{0}^{d}$, and get the following trivial proposition.

Proposition 2 : Given $x, y \in \mathbf{C}_{0}^{d}$, we have $N_{k} \partial_{e_{k}}=\partial_{e_{k}}\left(N_{k}-I\right)$ and $N_{k} \mu_{e_{k}}=\mu_{e_{k}}\left(N_{k}+I\right)$ on $\mathcal{A}_{0}\left(\mathbf{D}^{d}\right)$. 
For $x \in C_{0}^{d}$ we define the operators of creation $\mathbf{a}_{D}^{+}(x)$ and annihilation $\mathrm{a}_{D}(x)$ on $\mathcal{A}_{0}\left(D^{d}\right)$ setting

$$
\mathbf{a}_{D}^{+}\left(e_{k}\right)=\left(N_{k}+I\right)^{\frac{1}{2}} \mu_{e_{k}}
$$

and

$$
\mathbf{a}_{D}\left(e_{k}\right)=\partial_{e_{k}}\left(N_{k}+I\right)^{-\frac{1}{2}} .
$$

Proposition 3 : For $x, y \in \mathbf{C}_{0}^{d}$ the identitics

$$
\left[\mathbf{a}_{D}(x), \mathbf{a}_{D}^{+-}(y)\right]=\langle x, y\rangle I
$$

and

$$
\left[\mathbf{a}_{D}^{+}(x), \mathbf{a}_{D}^{+}(y)\right]=\left[\mathbf{a}_{D}(x), \mathbf{a}_{D}(y)\right]=0
$$

hold on $\mathcal{A}_{0}\left(\mathrm{D}^{d}\right)$.

Proof. Follows trivially from Proposition ?? and Lemma ??.

We write $\emptyset_{D}=1$ for the vacuum in $\mathcal{A}_{0}\left(D^{d}\right)$ and notice that $\mathbf{a}_{D}(x) \emptyset_{D}=$ 0 for all $x \in C_{0}^{d}$. In section 3 it will be clear, that $\mathcal{A}_{0}\left(D^{d}\right)$ now fulfils all the requirements for a Bose-Fock algebra, with the exception of $\mathbf{a}_{D}(x)$ and $\mathbf{a}_{D}^{+}(x)$ being dual operators. The proof of this is a little intricate and will be postponed to proposition??

Define

$$
\begin{aligned}
\varepsilon_{\underline{n}} & =\frac{1}{\sqrt{n_{1} !}}\left(\mathrm{a}_{D}^{+}\left(e_{1}\right)\right)^{n_{1}} \frac{1}{\sqrt{n_{2} !}}\left(\mathrm{a}_{D}^{+}\left(e_{2}\right)\right)^{n_{2}} \cdots \emptyset_{D} \\
& =\prod_{k=1}^{d}\left(\sqrt{\left(n_{k}+1\right)}\left\langle\cdot, e_{k}\right\rangle^{n_{k}}\right) .
\end{aligned}
$$

It is obvious that $\left\{\varepsilon_{\underline{n}}\right\}_{\underline{n} \in I}$ spans $\mathcal{A}_{0}\left(D^{d}\right)$, where

$$
I=\left\{\left(n_{1}, n_{2}, \ldots\right) \in R^{d} \mid n_{i} \in N_{0}, n_{i}=0 \text { for almost all } i \in N_{d}\right\} .
$$

Proposition $4:\left\{\varepsilon_{\underline{n}}\right\}_{\underline{n} \in I}$ isanorthonormalbasisin $\mathrm{A}_{0}\left(\mathbf{D}^{d}\right)$. 
Proof. We use the notation $\delta_{\underline{m}, \underline{n}}=\prod_{p=1}^{d} \delta_{m_{p}, n_{p}}$. Let $z_{p}=r_{p} e^{i \phi_{p}}, R_{p}=r_{p}^{2}$ and $z=\sum_{p=1}^{d} z_{p} e_{p}$, and write briefly

$$
\int_{D} f\left(R_{p}, \phi_{p}\right) \equiv \frac{1}{2 \pi} \int_{0}^{1} \int_{0}^{2 \pi} f\left(R_{p}, \phi_{p}\right) d \phi_{p} d R_{p}
$$

Then

$$
\left\langle z, e_{p}\right\rangle^{n_{p}}=\left\langle z_{p} e_{p}, e_{p}\right\rangle^{n_{p}}={\overline{z_{p}}}^{n_{p}}=r_{p}^{n_{p}} e^{-i n_{p} \phi_{p}}
$$

such that

$$
\begin{aligned}
\left\langle\prod_{i=1}^{d}\left\langle\cdot, e_{i}\right\rangle^{m_{i}}, \prod_{j=1}^{d}\left\langle\cdot, e_{j}\right\rangle^{n_{j}}\right\rangle_{2} & =\int_{D^{d}} \overline{\prod_{i=1}^{d}\left\langle\cdot, e_{i}\right\rangle^{m_{i}}} \prod_{j=1}^{d}\left\langle\cdot, e_{j}\right\rangle^{n_{j}} \\
& =\prod_{k=1}^{d} \int_{D} \overline{\left\langle\cdot, e_{k}\right\rangle^{m_{k}}}\left\langle\cdot, e_{k}\right\rangle^{n_{j}} \\
& =\prod_{p=1}^{d} \frac{1}{2 \pi} \int_{0}^{1} \int_{0}^{2 \pi} r_{p}^{m_{p}+n_{p}} e^{i\left(m_{p}-n_{p}\right) \phi_{p}} d \phi_{p} d R_{p} \\
& =\delta_{\underline{n}, \underline{m}}\left(\prod_{p=1}^{d} \frac{1}{n_{p}+1}\right),
\end{aligned}
$$

and hence

$$
\left\langle\varepsilon_{\underline{m}}, \varepsilon_{\underline{n}}\right\rangle_{2}=\delta_{\underline{n}, \underline{m}}
$$

follows.

\section{Introduction to Bose-Fock space}

To a given separable complex Hilbert space $\mathcal{H},\langle\cdot, \cdot\rangle$ attach a commutative algebra $\Gamma_{0} \mathcal{H}$ generated by $\mathcal{H}$ (one-particle space) and a unity which will be denoted by $\emptyset$ and called the vacuum. We call $\Gamma_{0} \mathcal{H}$ a Bose-Fock algebra (cf. [2], [3] and [5]) if

1. The scalar product from $\mathcal{H}$ extends over $\Gamma_{0} \mathcal{H}$ in such a way that for every $x \in \mathcal{H}$ the operator $\mathbf{a}^{+}(x)$ of multiplication by $x$ admits the adjoint $\mathbf{a}(x)$ defined on the whole $\Gamma_{0} \mathcal{H}$.

2. The adjoint fulfils the Leibniz rule, i.e. for every $x, y \in \mathcal{H}$ and $f, g \in$ $\Gamma_{0} \mathcal{H}$

$$
\langle x f, g\rangle=\langle f, \mathbf{a}(x) g\rangle
$$




$$
\left[\mathbf{a}(x), \mathbf{a}^{+}(y)\right]=\langle x, y\rangle I
$$

on $\Gamma_{0} \mathcal{H}$, and

$$
\mathbf{a}(x) \varnothing=0 .
$$

The operators $\mathbf{a}^{+}(x)$ (respectively $\mathbf{a}(x)$ ) will be called creations (respectively annihilations).

We define $\Gamma \mathcal{H}$ as the limits with respect to $|\cdot|$ (where $|x|=\langle x, x\rangle^{\frac{1}{2}}$ ) of all Cauchy sequences $\left\{g_{n}\right\} \subset \Gamma_{0} \mathcal{H}$. The Hilbert space $\Gamma \mathcal{H}$ will be called the Bose-Fock space.

Let $\left\{e_{i}\right\}_{i=1}^{d}$ be an orthonormal basis in $\mathcal{H}$ and $\mathcal{D}=\operatorname{span}_{n \in N_{d}}\left\{e_{n}\right\}$. Though $\mathcal{D}$ is not a Hilbert space, we can still consider $\Gamma_{0} \mathcal{D}$, and will call $\Gamma_{0} \mathcal{D}$ a Bose-Fock algebra. If $\mathcal{H}$ is finite dimensional, then $\Gamma_{0} \mathcal{D}$ obviously equals $\Gamma_{0} \mathcal{H}$. In any case the completion $\Gamma \mathcal{D}$ equals $\Gamma \mathcal{H}$.

We shall identify $\mathcal{D}$ with $C_{0}^{d}$ by use of the transformation

$$
\mathcal{D} \ni x \rightarrow \underline{x}=\left(x_{1}, x_{2}, \ldots\right) \in C_{0}^{d},
$$

where $x_{n}=\left\langle e_{n}, x\right\rangle, n \in N_{d}$. This identification will also be used for extensions of $\mathcal{D}$ and $C_{0}^{d}$.

We consider on $\Gamma_{0} \mathcal{H}$ the number operator

$$
\mathcal{N}=\sum_{p=1}^{\infty} \mathbf{a}^{+}\left(e_{p}\right) \mathbf{a}\left(e_{p}\right),
$$

characterised by the property that $\mathcal{N} x_{1} \cdots x_{m}=m x_{1} \cdots x_{m}$, for $x_{1}, \ldots, x_{m} \in$ $\mathcal{H}$. Furthermore the set $\left\{e_{\underline{n}}\right\}_{\underline{n} \in I}$ is an orthonormal basis for $\Gamma_{0} \mathcal{H}$, where

$$
e_{\underline{n}}=\frac{1}{\sqrt{n_{1} !}}\left(\mathbf{a}^{+}\left(e_{1}\right)\right)^{n_{1}} \cdots \frac{1}{\sqrt{n_{d} !}}\left(\mathbf{a}^{+}\left(e_{d}\right)\right)^{n_{d}} \emptyset .
$$

Following [3], we introduce the complex wave-representation assigning to $f \in \Gamma \mathcal{H}$ the function $f[\cdot]$, where

$$
f[z]=\left\langle e^{z}, f\right\rangle, z \in \mathcal{H} .
$$

For $f, g \in \Gamma_{0} \mathcal{H}$ and $z \in \mathcal{H}$ we have

$$
(f \cdot g)[z]=f[z] \cdot g[z] .
$$




\section{Bose-Fock space on the polydisc}

In this section we shall produce the unitary isomorphism $U$ from $\Gamma_{0} \mathcal{H}$ onto $\mathcal{A}_{0}\left(D^{d}\right)$. Notice that

$$
\left(\prod_{k=1}^{\infty}\left(\mathcal{N}_{k}+I\right) !\right)^{\frac{1}{2}} e_{1}^{n_{1}} \cdots e_{m}^{n_{m}} \cdots=\left(\prod_{k=1}^{\infty}\left(n_{k}+1\right) !\right)^{\frac{1}{2}} e_{1}^{n_{1}} \cdots e_{m}^{n_{m}} \cdots
$$

holds, where

$$
\mathcal{N}_{k}=\mathbf{a}^{+}\left(e_{k}\right) \mathbf{a}\left(e_{k}\right) .
$$

Setting $k_{z}=\left(\prod_{k=1}^{\infty}\left(\mathcal{N}_{k}+I\right) !\right)^{\frac{1}{2}} e^{z}$ for $\underline{z} \in \ell_{2} \cap D^{d}$, we get

$$
\begin{aligned}
\left|k_{z}\right|^{2} & =\left|\left(\prod_{k=1}^{d}\left(\mathcal{N}_{k}+I\right) !\right)^{\frac{1}{2}} e^{z}\right|^{2} \\
& =\left|\prod_{k=1}^{d}\left(\left(\mathcal{N}_{k}+I\right) ! !^{\frac{1}{2}} e^{z_{k}}\right)\right|^{2} \\
& =\prod_{k=1}^{d}\left(\sum_{n=0}^{\infty}(n+1)\left\langle z_{k}, z_{k}\right\rangle^{n}\right) \\
& =\prod_{k=1}^{d}\left(1-\left|z_{k}\right|^{2}\right)^{-2},
\end{aligned}
$$

where the last equation follows from the Taylor expansion. Hence $k_{z} \in \Gamma \mathcal{H}$, for $\underline{z} \in \ell_{2} \cap D^{d}$.

If $\mathcal{H}$ is finite dimensional, we have $D^{d} \subset \ell_{2}$ and thus $k_{z} \in \Gamma \mathcal{H}$ for $\underline{z} \in D^{d}$.

For pairs $(f, z)$ where either $f \in \Gamma \mathcal{H}$ and $\underline{z} \in \ell_{2} \cap D^{d}$ or $f \in \Gamma_{0} \mathcal{D}$ and $\underline{z} \in D^{d}$, we define the linear operator $U$ setting

$$
(U f)(\underline{z})=\left\langle k_{z}, f\right\rangle .
$$

It is clear that in the first case $(U f): \ell_{2} \cap D^{d} \rightarrow C$, and in the second case $(U f): D^{d} \rightarrow C$. We have $U: \Gamma_{0} \mathcal{D} \rightarrow \mathcal{A}_{0}\left(D^{d}\right)$.

Moreover, for $k \in N_{d}$

$$
U e_{k}^{n}=(n+1) ! \frac{1}{2}\left(e_{k}^{n}\right)[\cdot],
$$


and

$$
U \emptyset=1=\emptyset_{D} .
$$

Lemma 5 : The operator $U: \Gamma_{0} D \rightarrow \mathcal{A}_{0}\left(\mathbf{D}^{d}\right)$ is an isometry.

Proof. Take the orthonormal basis $\left\{e_{n}\right\}$ in $\mathcal{D}$ and let $e_{\underline{n}}$ be as defined in section 3. Then

$$
\begin{aligned}
\left(U e_{\underline{n}}\right)(\underline{z}) & =\left\langle\left(\prod_{k=1}^{\infty}\left(\mathcal{N}_{k}+I\right) !\right)^{\frac{1}{2}} e^{z}, e_{\underline{n}}\right\rangle \\
& =\left(\prod_{k=1}^{\infty} \sqrt{\left(n_{k}+1\right) !} \frac{1}{\sqrt{n_{k} !}}\left\langle z, e_{k}\right\rangle^{n_{k}}\right) \\
& =\left(\prod_{k=1}^{\infty} \sqrt{\left(n_{k}+1\right)}\left\langle\underline{z}, \underline{e_{k}}\right\rangle^{n_{k}}\right) \\
& =\varepsilon_{\underline{n}}(\underline{z}) .
\end{aligned}
$$

and thus $U: \Gamma_{0} \mathcal{D} \rightarrow \mathcal{A}_{0}\left(D^{d}\right)$ takes an orthonormal basis into an orthonormal basis, and hence is isometric on $\Gamma_{0} \mathcal{D}$.

Lemma 6 : Let $\mathcal{H}$ be finite dimensional and take $\left\{f_{n}\right\} \subset \Gamma \mathcal{H}$. If $f_{n} \rightarrow 0$, then $\left(U f_{n}\right)(\underline{z}) \rightarrow 0$ for every $\underline{z} \in \mathcal{D}^{d}$.

Proof. Let $\underline{z} \in D^{d}$ be given. If $\mathcal{H}$ is finite dimensional, then $k_{z} \in \Gamma \mathcal{H}$. For $f \in \Gamma \mathcal{H}$ we have $|(U f)(\underline{z})|=\left|\left\langle k_{z}, f\right\rangle\right| \leq\left|k_{z}\right||f|$, and hence $\left(U f_{n}\right)(\underline{z}) \rightarrow$ 0 for $f_{n} \rightarrow 0$.

Theorem 7 : Let $\mathcal{H}$ be finite dimensional. Then the operator $U$ : $\Gamma \mathcal{H} \rightarrow \mathcal{A}\left(\mathbf{D}^{d}\right)$ is unitary.

Proof. It follows from Lemma ??, that $U$ is an isometric mapping of $\Gamma_{0} \mathcal{H}$ onto $\mathcal{A}_{0}\left(D^{d}\right)$. Let $\left\{f_{n}\right\} \subset \Gamma_{0} \mathcal{H}$ be a sequence converging to $f \in \Gamma \mathcal{H}$. Then $g_{n}=U f_{n}$ is a Cauchy sequence and $g_{n} \rightarrow g$ in $\mathcal{A}\left(D^{d}\right)$. Since $\mathcal{H}$ is 
finite dimensional it follows from Lemma ??, that $g_{n}$ converges pointwise to $U f$. Hence $g=U f$ a.e. and $U f \in \mathcal{A}\left(D^{d}\right)$.

If $\mathcal{H}$ is infinite dimensional, we write $U: \Gamma \mathcal{H} \rightarrow \mathcal{A}\left(D^{d}\right)$ for the unitary extension of $U: \Gamma_{0} \mathcal{D} \rightarrow \mathcal{A}_{0}\left(D^{d}\right)$.

We have thus produced an isomorphism $U: \Gamma \mathcal{H} \rightarrow \mathcal{A}\left(D^{d}\right)$, such that the Bergman Bose-Fock space $\mathcal{A}\left(D^{d}\right)$ and the abstract Bose-Fock space $\Gamma \mathcal{H}$ are isomorphic. Further we have that $\mathcal{A}_{0}\left(D^{d}\right)$ is the Bergman Bose-Fock algebra generated by the space of anti-holomorphic functions $\left\{\langle\cdot, \underline{x}\rangle \mid \underline{x} \in C_{0}^{d}\right\},\langle\cdot, \cdot\rangle_{2}$. The operators $\mathbf{a}_{D}^{+}(\underline{x})$ (respectively $\mathbf{a}_{D}(\underline{x})$ ) are the operators of creation (respectively annihilation) and $\emptyset_{D}=1$ is a unit vector.

Proposition 8 : Let $\underline{x} \in \mathbf{C}_{0}^{d}$. We have $U \mathcal{N}_{k}=N_{k} U, U \mathbf{a}^{+}(x)=$ $\mathbf{a}_{D}^{+}(\underline{x}) U$ and $U \mathbf{a}(x)=\mathbf{a}_{D}(\underline{x}) U$ on $\Gamma_{0} \mathcal{D}$. If $f, g \in \mathcal{A}_{0}\left(\mathbf{D}^{d}\right)$ then $\left\langle\mathbf{a}_{D}(\underline{x}) f, g\right\rangle_{2}=$ $\left\langle f, \mathbf{a}_{D}^{+}(\underline{x}) g\right\rangle_{2}$.

Proof. Let $\underline{z} \in D^{d}$ and $f=\sum_{n=0}^{N} f_{n} \in \Gamma_{0} \mathcal{D}$, where $\mathcal{N}_{k} f_{n}=n f_{n}$. Then

$$
\begin{aligned}
\left(U \mathcal{N}_{k} f_{n}\right)(\underline{x}) & =\left\langle k_{z}, \mathcal{N}_{k} f_{n}\right\rangle=n\left\langle\left(\prod_{k=1}^{d}\left(\mathcal{N}_{k}+I\right) !\right)^{\frac{1}{2}} e^{z}, f_{n}\right\rangle \\
& =n\left(U f_{n}\right)(z)=\left(N_{k} U f_{n}\right)(z)
\end{aligned}
$$

and we have $U \mathcal{N}_{k}=N_{k} U$ on $\Gamma_{0} \mathcal{D}$.

Let $f \in \Gamma_{0} \mathcal{D}$ and $\underline{z} \in D^{d}$. Then

$$
\begin{array}{ccc}
\left(U \mathbf{a}^{+}\left(e_{n}\right) f\right)(\underline{z})=\left\langle k_{z}, \mathbf{a}^{+}\left(e_{n}\right) f\right\rangle=\left\langle\mathbf{a}\left(e_{n}\right)\left(\prod_{k=1}^{d}\left(\mathcal{N}_{k}+I\right) !\right)^{\frac{1}{2}} e^{z}, f\right\rangle \\
= & \left\langle\left(\mathcal{N}_{n}+2 \cdot I\right) ! \frac{1}{2}\left(\prod_{k=1, k \neq n}^{d}\left(\mathcal{N}_{k}+I\right) !\right)^{\frac{1}{2}} \mathbf{a}\left(e_{n}\right) e^{z}, f\right\rangle \\
= & \left\langle\underline{z}, \underline{e_{n}}\right\rangle\left\langle\left(\prod_{k=1}^{d}\left(\mathcal{N}_{k}+I\right) !\right)^{\frac{1}{2}} e^{z},\left(\mathcal{N}_{n}+2 \cdot I\right)^{\frac{1}{2}} f\right\rangle \\
= & \mu_{\underline{e_{n}}}\left(U\left(\mathcal{N}_{n}+2 \cdot I\right)^{\frac{1}{2}} f\right)(\underline{z})=\mu_{\underline{e_{n}}}\left(\mathcal{N}_{n}+2 \cdot I\right)^{\frac{1}{2}}(U f)(\underline{z}) \\
= & \left(\mathcal{N}_{n}+I\right)^{\frac{1}{2}} \mu_{\underline{e_{n}}}(U f)(\underline{z})=\left(\mathbf{a}_{D}^{+}\left(\underline{e_{n}}\right) U f\right)(\underline{z}),
\end{array}
$$

and thus $U \mathbf{a}^{+}(x)=\mathbf{a}_{D}^{+}(\underline{x}) U$ on $\Gamma_{0} \mathcal{D}$. Moreover 


$$
\begin{aligned}
\left(U \mathbf{a}\left(e_{n}\right) f\right)(z) & =\left\langle k_{z}, \mathbf{a}\left(e_{n}\right) f\right\rangle=\left\langle\mathbf{a}^{+}\left(e_{n}\right)\left(\prod_{k=1}^{d}\left(\mathcal{N}_{k}+I\right) !\right)^{\frac{1}{2}} e^{z}, f\right\rangle \\
& =\left\langle e_{n} e^{z},\left(\prod_{k=1, k \neq n}^{d}\left(\mathcal{N}_{k}+I\right) !\right)^{\frac{1}{2}} \mathcal{N}_{n} ! \frac{1}{2} f\right\rangle \\
& =\partial_{\underline{e_{n}}}\left\langle e^{z},\left(\prod_{k=1}^{d}\left(\mathcal{N}_{k}+I\right) !\right)^{\frac{1}{2}}\left(\mathcal{N}_{n}+I\right)^{-\frac{1}{2}} f\right\rangle \\
& =\left(\partial_{\underline{e_{n}}} U\left(\mathcal{N}_{n}+I\right)^{-\frac{1}{2}} f\right)(\underline{z}) \\
& =\left(\partial_{\underline{e_{n}}}\left(N_{n}+I\right)^{-\frac{1}{2}} U f\right)(\underline{z})=\left(\mathbf{a}_{D}\left(\underline{e_{n}}\right) U f\right)(\underline{z}),
\end{aligned}
$$

and thus $U \mathbf{a}(x)=\mathbf{a}_{D}(\underline{x}) U$ on $\Gamma_{0} \mathcal{D}$. Furthermore

$$
\begin{aligned}
\left\langle\mathbf{a}_{D}(\underline{x}) f, g\right\rangle_{2} & =\left\langle U^{-1} \mathbf{a}_{D}(\underline{x}) f, U^{-1} g\right\rangle=\left\langle\mathbf{a}(x) U^{-1} f, U^{-1} g\right\rangle \\
& =\left\langle U^{-1} f, \mathbf{a}^{+}(x) U^{-1} g\right\rangle=\left\langle U^{-1} f, U^{-1} \mathbf{a}_{D}^{+}(\underline{x}) g\right\rangle \\
& =\left\langle f, \mathbf{a}_{D}^{+}(\underline{x}) g\right\rangle_{2} .
\end{aligned}
$$

for $f, g \in \mathcal{A}_{0}\left(D^{d}\right)$.

Notice that on $\Gamma_{0} \mathcal{D}$ we have that $\mathbf{a}_{D}(\underline{x})$ is the adjoint operator of $\mathbf{a}_{D}^{+}(\underline{x})$.

We conclude that $\mathcal{A}_{0}\left(D^{d}\right)$ is the Bose-Fock algebra generated by the Hilbert space of $L^{2}$-integrable anti-holomorphic functions $\left\{\langle\cdot, \underline{x}\rangle \mid \underline{x} \in C_{0}^{d}\right\},\langle\cdot, \cdot\rangle_{2}$. The operators $\mathbf{a}_{D}^{+}(\underline{x})$ (respectively $\left.\mathbf{a}_{D}(\underline{x})\right)$ are the operators of creation (respectively annihilation) and $\emptyset_{D}=1$ is a unit vector.

Let $\mathcal{H}$ be finite dimensional and $\underline{\lambda} \in D^{d}$. Since

$$
(U f)(\underline{\lambda})=\left\langle k_{\lambda}, f\right\rangle=\left\langle U k_{\lambda}, U f\right\rangle_{2}
$$

holds for $f \in \Gamma_{0} \mathcal{H}$, the function $\left(U k_{\lambda}\right)(\cdot)$ on $D^{d}$ is the reproducing kernel in $\mathcal{A}\left(D^{d}\right)$, and for $\underline{z} \in D^{d}$ wo have

$$
\left(U k_{\lambda}\right)(\underline{z})=\left(U\left(\prod_{k=1}^{d}\left(\mathcal{N}_{k}+I\right) !\right)^{\frac{1}{2}} e^{\mathrm{a}^{\digamma}(\lambda)} \phi\right)(\underline{z})
$$




$$
\begin{aligned}
& =\left(\left(\prod_{k=1}^{d}\left(N_{k}+I\right) !\right)^{\frac{1}{2}} e^{\sum_{n=1}^{d} \mathbf{a}_{D}^{+}\left(\lambda_{n} \underline{e_{n}}\right)} U \emptyset\right)(\underline{z}) \\
& =\prod_{k=1}^{d}\left(\left(N_{k}+I\right) ! e^{\left\langle z_{k}, \lambda_{k}\right\rangle}\right) \\
& =\prod_{k=1}^{d}\left(\sum_{n=0}^{\infty}(n+I)\left(\overline{z_{k}} \lambda_{k}\right)^{n}\right) \\
& =\prod_{k=1}^{d}\left(\left(1-\overline{z_{k}} \lambda_{k}\right)^{-2}\right) .
\end{aligned}
$$

Hence $\left(U k_{\lambda}\right)(\cdot)$ is the Bergman kernel for $L^{2}\left(D^{d}\right)$.

\section{References}

[1] B.C. Hall, The Segal-Bargmann "Coherent State" Transform for Compact Lie Groups, J. Funct. Anal. 122 (1994) 103-151.

[2] E. Nelson, Probability theory and euclidean field theory, Lecture Notes in Phys. 25 (1973), 94-124, Springer-Verlag.

[3] T.T. Nielsen, Bose Algebras: The Complex and Real Wave Representations, Lecture Notes in Math. 1472 (1991), Springer-Verlag.

[4] K. Seip, Reproducing formulas and double orthogonality in Bargmann and Bergman spaces, Siam J. Math. Anal., Vol. 22, No. 3, (1991) 856876.

[5] W. Slowikowski, Infinite dimensional Lie algebras and Lie groups of operators for paired quantum particles, to appear. slowi@mi.aau.dk.

Received : September 1997. 
Olé Rask

Instituto de Matemáticas

Universidad Austral de Chile

Casilla 567

Valdivia 